\title{
Effect of Mirror Misalignments on Optical Ray Path In a Ring Resonator
}

\author{
Dong-Chan Lee*, Jae-Cheul Lee, Seong-Hyun Son, and Hyun-Ju Cho \\ Center for Photonics \& Communications, Institute for Advanced Engineering, \\ Kyonggi-do 449-860, KOREA
}

(Received March 18, 2002)

\begin{abstract}
The operating principal of a ring laser gyroscope depends on the phase difference for the counterpropagating waves within a closed path. The reflecting mirrors mounted on the monoblock form the traveling waves. The manufacturing accuracy of the monoblock influences the traveling path of ray, the sensitivity of laser resonator for misalignments, and diffraction losses.

A $3 \times 3$ ray transfer matrix was derived for optical components with centering and squaring errors in a ring resonator. The matrix can be utilized to predict the optical ray paths on the basis of the manufacturing errors of the monoblock as well as the misalignment of mirrors. Then the distance and orientation (or slope) at the arbitrary plane inside the resonator along the ideal optical path can be calculated from the chain multiplication of the ray transfer matrix for each optical component in one round trip. We also show that the counter-propagating rays in a ring resonator with errors does not coincide in each round trip, which results in gain difference between two beams, and how these errors can be adjusted through the alignment procedure. Finally this $3 \times 3$ ray matrix formalism can be used to calculate the beam size and its displacement from the optical axis and the deviation at the diaphragm.
\end{abstract}

OCIS codes : $080.2740,200.0210,220.0220,220.4830,220.4880$.

\section{INTRODUCTION}

One of the most expensive and difficult parts in the ring laser gyroscope is to manufacture a monoblock (in order words, one body block), where the mirrors are mounted to form a resonator. The manufacturing accuracy of the monoblock influences the optical beam path in the resonator, misalignments sensitivity, and diffraction losses. The deviations of the angle between mirror mounted planes, the mismatch of cross points of channel axes with planes, and the deviations of the angle between the axes of channel and planes are the errors in manufacturing monoblocks. In an ideal case, the ray path in a resonator coincides with the reference optical axis. However, in fact, misaligned elements with centering and squaring error exist. The effective optical axis is changed in a zigzag fashion, shifting or bending from plane to plane within the periodic round trip. Moreover, the ray axis propagating clockwise and counter-clockwise may not lie on the same path. Here a ray is defined as the path that the center of a very slowly diverging electromagnetic beam would take as it goes through the system.
In 1969, Ishchenko presented the geometrical descriptions of an optical path due to the mirror misalignments in a ring resonator, which results from the imperfect construction and mounting of the optical elements [1]. His description helps one understand the misalignment effects of elements in the ray path. However, since the geometrical descriptions need to change for the various optical mounting systems, the sequential derivations of ray path are difficult.

The ray transfer matrix [2] can be utilized in order to solve this difficulty in a concrete way. We introduce $3 \times 3$ matrix formalism in tracing the ray path along the optical components with centering and squaring errors and show how this can be used to calculate the displacement and orientation in a closed optical system. Then the $3 \times 3$ matrix for the optical components such as a curved mirror with tilt and displacement is derived in a ring resonator with 4 mirrors, as an example. These matrices are multiplied in sequence as they are encountered, until one round trip is made. Depending on the starting reference plane in the path, this total matrix is different, and, it is used to calculate the displacement and slope at the plane, 
and the beam size. The simulation result will be given for a square ring resonator with the total length of 16 $\mathrm{cm}$.

\section{MATRIX REPRESENTATION OF AN OPTICAL SYSTEM WITH MISALIGNMENTS}

In an ideal optical system, the $2 \times 2$ ray matrix as shown in Fig. 1 is used to represent input-output characteristics in the displacement and orientation (or slope) of a ray, which assumes that all the paraxial elements are properly aligned and centered with respect to optical axis [3].

$$
\left\{\begin{array}{c}
X_{i+1} \\
\phi_{i+1}
\end{array}\right\}=\left[\begin{array}{cc}
A_{i} & B_{i} \\
C_{i} & D_{i}
\end{array}\right]\left\{\begin{array}{c}
X_{i} \\
\phi_{i}
\end{array}\right\}
$$

For example, in free space propagation of interval (or distance) $L, A=1, B=L, C=0$, and $D=1$.

In a real system, however, the position of the optical axis may be slightly displaced from its assumed position by a small distance $\Delta X_{1}$, and the exact direction in which the optical axis is pointing deviates slightly from its assumed direction by a small angle $\Delta \phi_{i}[2]$. This effect can occur if one of the optical components has not been properly centered. Under these circumstances, the $3 \times 3$ ray transfer matrix can be introduced as shown in Eq. (2),

$$
\left\{\begin{array}{c}
X_{i+1} \\
\phi_{i+1} \\
1
\end{array}\right\}=\left[\begin{array}{ccc}
A_{i} & B_{i} & \Delta X_{i} \\
C_{i} & D_{i} & \Delta \phi_{i} \\
0 & 0 & 1
\end{array}\right]\left\{\begin{array}{c}
X_{i} \\
\phi_{i} \\
1
\end{array}\right\}=M_{i}\left\{\begin{array}{c}
X_{i} \\
\phi_{i} \\
1
\end{array}\right\}
$$

Now consider an optical system for which the multiplication of the transfer matrix gives the overall augmented matrix, $M t_{m}$

$$
M t_{m}=M_{m} M_{m-1} \cdots M_{i} \cdots M_{3} M_{2} M_{1}
$$

where $M_{i}=\left[\begin{array}{ccc}A_{i} & B_{i} & \Delta X_{i} \\ C_{i} & D_{i} & \Delta \phi_{i} \\ 0 & 0 & 1\end{array}\right]$ is the unimodular matrix representing transfer from $i^{\text {th }}$ intermediate reference plane to the $(i+1)^{t h}$. If we assume that the overall augmented matrix $M t_{n}$ represents the effect of a single round trip in an optical resonator, due to its repeatability, it can be written by,

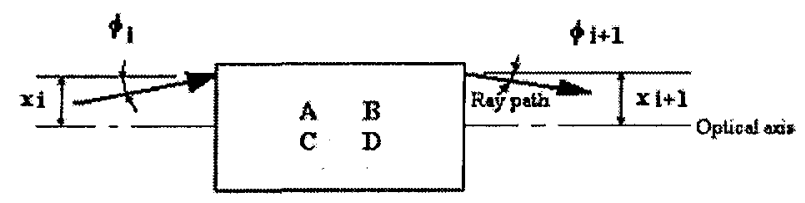

FIG. 1. Ray matrix or ABCD matrix in an aligned optical system.

$$
\left\{\begin{array}{c}
X_{n} \\
\phi_{n} \\
1
\end{array}\right\}=\left[\begin{array}{ccc}
A_{n} & B_{n} & \Delta X_{n} \\
C_{n} & D_{n} & \Delta \phi_{n} \\
0 & 0 & 1
\end{array}\right]\left\{\begin{array}{c}
X_{n} \\
\phi_{n} \\
1
\end{array}\right\}=M t_{n}\left\{\begin{array}{c}
X_{n} \\
\phi_{n} \\
1
\end{array}\right\}
$$

where $A_{n}, B_{n}, C_{n}, D_{n}, \Delta X_{n}$ and $\Delta \phi_{n}$ are the entries of $M t_{n}$ and $A_{n} D_{n}-B_{n} C_{n}=1$. From Eq. (4), the displacement and slope of an input ray can be calculated as follows,

$$
\begin{aligned}
X_{n} & =\frac{\left(1-D_{n}\right) \Delta X_{n}+B_{n} \Delta \phi_{n}}{\left(2-A_{n}-D_{n}\right)} \\
\phi_{n} & =\frac{C_{n} \Delta X_{n}+\left(1-A_{n}\right) \Delta \phi_{n}}{\left(2-A_{n}-D_{n}\right)}
\end{aligned}
$$

\section{RAY MATRIX FOR OPTICAL COMPONENTS WITH MISALIGNMENT IN A RING RESONATOR}

Let us consider a ring resonator as shown in Fig. 2, which consists of 4 mirrors M1, M2, M3, and M4. In a typical ring laser gyroscope, two flats (M1, M4) and two spherical mirrors (M2, M3) are used. The distance between two adjacent mirrors along the beam path is $L$. The mirrors are attached to the monoblock (in other words, one-body block) made of Zerodur ${ }^{\mathrm{TM}}$, which has the lowest thermal expansion coefficient. This monoblock has a shape of square with corner edge cut-off of small prisms to allow mirror attachment. The channels between the mirrors are bored to have a space for beam propagation. The manufacturing tolerance of this monoblock is very important

(a)

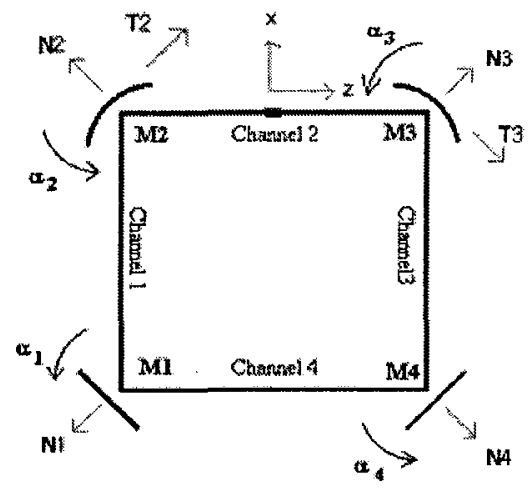

(b)

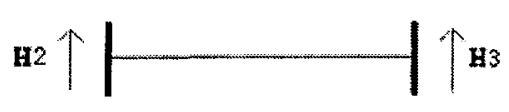

FIG. 2. Geometrical parameters of ring laser monoblock and its configuration. 


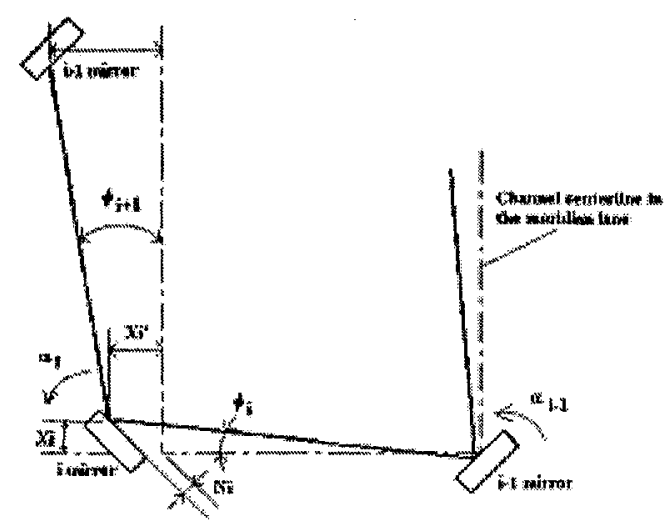

FIG. 3. Schematic drawing for the ray optical path $n$ the Meridian plane.

for a stable laser operation. However, in reality, there exist some manufacturing errors such as:

(1) Angular deviation from $45^{\circ}$ in the Meridian plane : $\alpha_{1}, \alpha_{2}, \alpha_{3}, \alpha_{4}$

(2) Angular deviation from $90^{\circ}$ in the Sagittal plane : $\beta_{1}, \beta_{2}, \beta_{3}, \beta_{4}$

(3) Mismatch of the cross points between channel axes: $N_{1}, N_{2}, N_{3}, N_{4}$

In handling an optical system with reflective optical components, it is important to keep the coordinate consistent. Fig. 2 shows how its right-handed coordinate system changes upon reflection from mirrors in a clockwise direction, where the beam propagates along the z-axis. The adjustments of the optical path are done by the movement of spherical mirrors 2 and 3 along the horizontal direction of $T_{2}, T_{3}$ and along the vertical direction of $\mathrm{H}_{2}, \mathrm{H}_{3}$.

Fig. 3 shows the optical beam path due to the misalignment. Let us calculate how the coordinate of the beam incident to the $i^{\text {th }}$ mirror changes in the Meridian plane. The propagation angle of beam $\phi_{i}$ is positive, if the beam propagates in the direction of increasing transverse coordinate. For the flat mirrors with $\alpha_{i}$, when the incidence angle is $\phi_{i}$ and the reflected angle becomes

$$
\phi_{i+1}=-\phi_{i}+2 \alpha_{i}
$$

If the coordinates of the incident beam $X_{i}$ onto $i^{\text {th }}$ mirror is negative and the coordinate of the reflected ray $X_{i}^{\prime}$ right after $i^{t h}$ mirror is positive, that is, $X_{i}^{\prime}=$ $-X_{i} . N_{i}$ is positive, if the cross point of centerlines of channels is outside the monoblock, and negative, if it appears inside the monoblock. With positive $N_{i}$, the coordinate of reflected beam increases by a factor of $\sqrt{2}$. When the beam propagates between $i^{\text {th }}$ and $(i+1)^{t h}$ mirror, its linear coordinates are transformed as follows

$$
X_{i+1}=-X_{i}+\phi_{i+1} L+\sqrt{2} N_{i}
$$

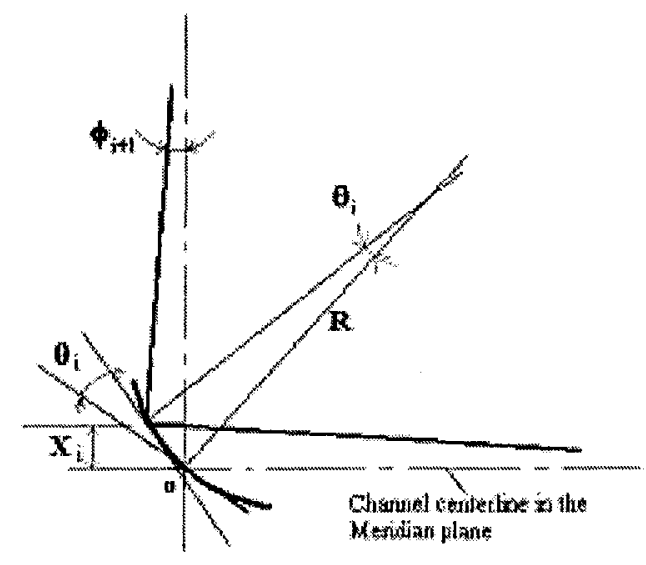

FIG. 4. Schematic drawing of angle coordinates on a spherical mirror.

For the spherical mirrors, the reflection angle depends on the coordinate of the incident ray on the mirror surface as shown in Fig. 4. Assume that the $i^{t h}$-spherical mirror of radius $R_{i}$ is set at $45^{\circ}$. The reflected beam $\phi_{i+1}$ from point $\mathrm{O}$ is $-\phi_{i}$. The reflection angle from the location of $X_{i}$ relative to the point $\mathrm{O}$ can be written as,

$$
\phi_{i+1}=2 \theta_{i}=\frac{2 \sqrt{2} X_{i}}{R_{i}}
$$

If one takes into account of $N_{i}$ and $T_{i}$, the angular coordinate of reflected beam could be written as,

$$
\phi_{i+1}=-\phi_{i}+2 \alpha_{i}+\frac{2 \sqrt{2}}{R_{i}}\left(X_{i}-\frac{N_{i}}{\sqrt{2}}+\frac{T_{i}}{\sqrt{2}}\right)
$$

Using Eqs. (9) and (6) can be rewritten in terms of the positions of the incident beam

$$
\begin{aligned}
X_{i+1} & =\left(\frac{2 \sqrt{2}}{R_{i}} L-1\right) X_{i}-\phi_{i} L \\
& +\left(2 \alpha_{i}-\frac{2}{R_{i}} N_{i}+\frac{2}{R_{i}} T_{i}\right) L+\sqrt{2} N_{i}
\end{aligned}
$$

Also, the positions and slopes in the Sagittal plane must be considered, this plane is in the vertical direction of Y. At the propagation between mirrors $i$ and $i+1$, if the angular coordinates of the ray change on the channels, its linear coordinates are given as,

$$
Y_{i+1}=Y_{i}+\psi_{i+1} L
$$

The propagation angle $\psi_{i}$ is positive, if the ray propagates in the direction of increasing the vertical coordinate. For flat mirrors with tilt $\beta_{i}$ from $90^{\circ}$, the reflection angle is 
TABLE 1. Ray matrices for optical components in a ring resonator (clockwise direction).

\begin{tabular}{|c|c|c|}
\hline & Meridian plane & Sagittal plane \\
\hline Interval matrix & {$\left[\begin{array}{lll}1 & L & 0 \\
0 & 1 & 0 \\
0 & 0 & 1\end{array}\right]$} & {$\left[\begin{array}{lll}1 & L & 0 \\
0 & 1 & 0 \\
0 & 0 & 1\end{array}\right]$} \\
\hline $\begin{array}{l}\text { Reflection matrix from } \\
\text { spherical mirror }\end{array}$ & $\begin{array}{ccc}-1 & 0 & \sqrt{2} N_{i} \\
\frac{2 \sqrt{2}}{R_{i}} & -1 & 2 \alpha_{i}-\frac{2}{R_{i}} N_{i}+\frac{2}{R_{i}} T_{i} \\
0 & 0 & 1\end{array}$ & $\begin{array}{ccc}1 & 0 & 0 \\
-\frac{\sqrt{2}}{R_{i}} & 1 & 2 \beta_{i}+\frac{\sqrt{2}}{R_{i}} H_{i} \\
0 & 0 & 1\end{array}$ \\
\hline $\begin{array}{l}\text { Reflection matrix from flat } \\
\text { mirror }\end{array}$ & $\begin{array}{ccc}-1 & 0 & \sqrt{2} N_{i} \\
0 & -1 & 2 \alpha_{i} \\
0 & 0 & 1\end{array}$ & {$\left[\begin{array}{ccc}1 & 0 & 0 \\
0 & 1 & 2 \beta_{i} \\
0 & 0 & 1\end{array}\right]$} \\
\hline
\end{tabular}

$$
\psi_{i+1}=\psi_{i}+2 \beta_{i}
$$

For the spherical mirrors, the reflection angle depends on the coordinate of the incident ray on the mirror surface. Assume that the $i^{t h}$-spherical mirror of radius $R_{i}$ is set at $45^{\circ}$. The reflected beam $\phi_{i+1}$ from point $\mathrm{O}$ is $-\phi_{i}$. In a similar way, the reflection angle from the location of $Y_{i}$ relative to the point $O$ can be written as,

$$
\psi_{i+1}=\psi_{i}-\frac{\sqrt{2}}{R_{i}} Y_{i}+2 \beta_{i}+\frac{\sqrt{2}}{R_{i}} H_{i}
$$

Using Eqs. (13) and (11) can be rewritten in terms of the positions of the incident ray,

$$
Y_{i+1}=\left(1-\frac{\sqrt{2}}{R_{i}} L\right) Y_{i}+\psi_{i} L+\left(2 \beta_{i}+\frac{\sqrt{2}}{R_{i}} H_{i}\right) L
$$

Eqs. (9), (10), (13), and (14) can be rewritten in a matrix form as follows.

$$
\begin{aligned}
& \left\{\begin{array}{c}
X_{i+1} \\
\phi_{i+1} \\
1
\end{array}\right\}=\left[\begin{array}{lll}
1 & L & 0 \\
0 & 1 & 0 \\
0 & 0 & 1
\end{array}\right]\left[\begin{array}{ccc}
-1 & 0 & \sqrt{2} N_{i} \\
\frac{2 \sqrt{2}}{R_{i}} & -1 & 2 \alpha_{i}-\frac{2}{R_{i}} N_{i}+\frac{2}{R_{i}} T_{i} \\
0 & 0 & 1
\end{array}\right]\left\{\begin{array}{c}
X_{i} \\
\phi_{i} \\
1
\end{array}\right\} \\
& \left\{\begin{array}{c}
Y_{i+1} \\
\psi_{i+1} \\
1
\end{array}\right\}=\left[\begin{array}{lll}
1 & L & 0 \\
0 & 1 & 0 \\
0 & 0 & 1
\end{array}\right]\left[\begin{array}{ccc}
1 & 0 & 0 \\
-\frac{\sqrt{2}}{R_{i}} & 1 & 2 \beta_{i}+\frac{\sqrt{2}}{R_{i}} H_{i} \\
0 & 0 & 1
\end{array}\right]\left\{\begin{array}{c}
Y_{i} \\
\psi_{i} \\
1
\end{array}\right\}
\end{aligned}
$$

Looking into the sequential ray matrices in Eq. (15), one can see that it consists of two consecutive ray matrices. One corresponds to the matrix due to free space propagation and the other for the reflection matrix of the spherical mirror. The characteristic ray matrices for optical elements in a ring resonator are summarized in Table 1. The flat mirror is a special case of $R_{i}=\infty$
In a ring resonator, the beam can travel to the opposite direction within a closed path. Thus, the optical path must be considered in the counter clockwise direction in Fig. 2. In a similar way, but considering its coordinate system, the sequential transfer matrices in the Meridian and Sagittal plane, can be rewritten as follows.

$$
\begin{aligned}
& \left\{\begin{array}{c}
X_{i-1} \\
\phi_{i-1} \\
1
\end{array}\right\}=\left[\begin{array}{lll}
1 & L & 0 \\
0 & 1 & 0 \\
0 & 0 & 1
\end{array}\right]\left[\begin{array}{ccc}
-1 & 0 & \sqrt{2} N_{i} \\
\frac{2 \sqrt{2}}{R_{i}} & -1 & 2 \alpha_{i+1}-\frac{2}{R_{i}} N_{i}+\frac{2}{R_{i}} T_{i} \\
0 & 0 & 1
\end{array}\right]\left\{\begin{array}{c}
X_{i} \\
\phi_{i} \\
1
\end{array}\right\} \\
& \left\{\begin{array}{c}
Y_{i-1} \\
\psi_{i-1} \\
1
\end{array}\right\}=\left[\begin{array}{lll}
1 & L & 0 \\
0 & 1 & 0 \\
0 & 0 & 1
\end{array}\right]\left[\begin{array}{ccc}
1 & 0 & 0 \\
-\frac{\sqrt{2}}{R_{i}} & 1 & 2 \beta_{i+1}+\frac{\sqrt{2}}{R_{i}} H_{i} \\
0 & 0 & 1
\end{array}\right]\left\{\begin{array}{c}
Y_{i} \\
\psi_{i} \\
1
\end{array}\right\}
\end{aligned}
$$


TABLE 2. Ray matrices for the optical components in a ring resonator (counter-clockwise direction).

\begin{tabular}{|c|c|c|}
\hline & Meridian plane & Sagittal plane \\
\hline Interval matrix & {$\left[\begin{array}{lll}1 & L & 0 \\
0 & 1 & 0 \\
0 & 0 & 1\end{array}\right]$} & {$\left[\begin{array}{lll}1 & L & 0 \\
0 & 1 & 0 \\
0 & 0 & 1\end{array}\right]$} \\
\hline $\begin{array}{l}\text { Reflection matrix from } \\
\text { spherical mirror }\end{array}$ & $\begin{array}{ccc}-1 & 0 & \sqrt{2} N_{i} \\
\frac{2 \sqrt{2}}{R_{i}} & -1 & 2 \alpha_{i+1}-\frac{2}{R_{i}} N_{i}+\frac{2}{R_{i}} T_{i} \\
0 & 0 & 1\end{array}$ & $\begin{array}{ccc}1 & 0 & 0 \\
-\frac{\sqrt{2}}{R_{i}} & 1 & 2 \beta_{i+1}+\frac{\sqrt{2}}{R_{i}} H_{i} \\
0 & 0 & 1\end{array}$ \\
\hline $\begin{array}{l}\text { Reflection matrix from flat } \\
\text { mirror }\end{array}$ & {$\left[\begin{array}{ccc}-1 & 0 & \sqrt{2} N_{i} \\
0 & -1 & 2 \alpha_{i+1} \\
0 & 0 & 1\end{array}\right.$} & {$\left[\begin{array}{ccc}1 & 0 & 0 \\
0 & 1 & 2 \beta_{i+1} \\
0 & 0 & 1\end{array}\right]$} \\
\hline
\end{tabular}

The sequential transfer matrices in Eq. (16) can be divided into the same characteristic transfer matrices as aforementioned. The characteristic matrices are given in Table 2.

Using the condition of Eqs. (3) and (4), the displacement and orientation on each mirror can be calculated. The overall augmented matrices for the ring resonator at each optical component is given by the sequential multiplication of each ray matrix from Tables 1 and 2, as in Eqs. (17) and (18).

For the clockwise direction,

$$
\begin{aligned}
& M t_{1}=M_{1} M_{0} M_{4} M_{0} M_{3} M_{0} M_{2} M_{0} \\
& M t_{2}=M_{2} M_{0} M_{1} M_{0} M_{4} M_{0} M_{3} M_{0} \\
& M t_{3}=M_{3} M_{0} M_{2} M_{0} M_{1} M_{0} M_{4} M_{0} \\
& M t_{4}=M_{4} M_{0} M_{3} M_{0} M_{2} M_{0} M_{1} M_{0}
\end{aligned}
$$

For the counter-clockwise direction,

$$
\begin{aligned}
& M t_{1}=M_{1} M_{0} M_{2} M_{0} M_{3} M_{0} M_{4} M_{0} \\
& M t_{2}=M_{2} M_{0} M_{3} M_{0} M_{4} M_{0} M_{1} M_{0} \\
& M t_{3}=M_{3} M_{0} M_{4} M_{0} M_{1} M_{0} M_{2} M_{0} \\
& M t_{4}=M_{4} M_{0} M_{1} M_{0} M_{2} M_{0} M_{3} M_{0}
\end{aligned}
$$

where $M_{0}$ describes the interval matrix for the free space propagation and $M_{i}$ describes the reflection matrix from the mirror (where $i=1,2,3,4$ ). Using Eq. (5) and the aforesaid overall augmented matrices, the displacements and orientations on each mirror, right after the mirror or just before the free propagation, can be described.

For $\mathrm{TEM}_{00}$ mode operation, a diaphragm filtering the various higher order modes is usually implemented in one channel as shown in Fig. 2. Typically it is located between two spherical mirrors or between two flat mirrors. If we assume that it locates between two spherical mirrors, the displacement and orientation at the diaphragm can be calculated from Eqs. (3) and (4).

$$
M t_{\text {diaphragm }}=M_{8} M_{2} M_{0} M_{1} M_{0} M_{4} M_{0} M_{3} M_{8}
$$

where $M_{8}$ is the interval matrix with $L / 2$.

And the spot size at the center of diaphragm [3] can be given by Eq. (20)

$$
\frac{\pi \omega^{2}}{\lambda}=\frac{B}{\left[1-\left(\frac{A+D}{2}\right)^{2}\right]^{1 / 2}}
$$

where $\omega$ is the beam spot size in the Meridian or Sagittal plane and $A, B, D$ are the entries in Eq. (19). Due to the astigmatism, the entries of the total matrix are different in both planes and obviously the beam spot has a elliptic shape .

\section{SIMULATION}

Using the ray transfer matrix in Tables 1 and 2, the ray trajectories are calculated for the monoblock with the geometrical parameters listed in Table 3 and its results are shown in Fig. 5. In this figure, the discontinuity from channel to channel comes from a coordinate system change upon reflection from the mirror. Actually there is no discontinuation. Note that the slopes on each channel and the effect of the sign of $\alpha$ are shown in the figure. One can also see two different paths for counter-propagating rays due to misalignment. From Fig. 5, the deviation at the center of the diaphragm (path length $60 \mathrm{~mm}$ from $\mathrm{M} 1$ ) in the Meridian plane is $0.035 \mathrm{~mm}$ in the clockwise direction and $0.04 \mathrm{~mm}$ in the counterclockwise direction. The spot size is $0.22 \mathrm{~mm}$. And the deviations from the diaphragm center in the Sagittal plane are approximately $0.128 \mathrm{~mm}$ in two directions. The spot size is $0.26 \mathrm{~mm}$. Fig. 6 shows the results after two spherical mirrors were adjusted to pass the center of the diaphragm. Note that $\mathrm{CW}$ beam cross the center at $z=60 \mathrm{~mm}$ as a result of alignments and the CCW beam is slightly off-centered. Maple V Release 5.1 was used for all the simulations

In order for the ray to pass through the center of diaphragm, the movement of spherical mirrors M2 and M3 with the same radius of curvature can generally be presented such as Eq. (21), using the ray transfer matrix Eq. (19). 
TABLE 3. Manufacturing tolerance of monoblock.

\begin{tabular}{|c|c|c|c|}
\hline No. & Parameter & Symbol & Parameter value \\
\hline 1 & Channel length (mm) & $L$ & 40 \\
\hline 2 & Radius of spherical mirror (mm) & $\tilde{R}\left(R_{2}, R_{3}\right)$ & 2000 \\
\hline \multirow{4}{*}{3} & \multirow{4}{*}{$\begin{array}{l}\text { Angular deviation from } 45^{\circ} \\
\text { in the Meridian plane (arc sec) }\end{array}$} & $\alpha_{1}$ & -30 \\
\hline & & $\alpha_{2}$ & 30 \\
\hline & & $\alpha_{3}$ & 30 \\
\hline & & $\alpha_{4}$ & -30 \\
\hline \multirow{4}{*}{4} & \multirow{4}{*}{$\begin{array}{l}\text { Angular deviation from } 90^{\circ} \\
\text { in the Sagittal plane (arc sec) }\end{array}$} & $\overline{\beta_{1}}$ & 5 \\
\hline & & $\beta_{2}$ & 5 \\
\hline & & $\beta_{3}$ & 5 \\
\hline & & $\beta_{4}$ & 5 \\
\hline \multirow{4}{*}{5} & \multirow{4}{*}{$\begin{array}{l}\text { Mismatch of the cross points } \\
\text { between channel axes ( } \mathrm{mm})\end{array}$} & $N_{1}$ & -0.05 \\
\hline & & $N_{2}$ & 0.05 \\
\hline & & $N_{3}$ & 0.05 \\
\hline & & $N_{4}$ & -0.05 \\
\hline
\end{tabular}

$$
\begin{gathered}
T 2=T 3-N 2-N 3+R\left(\alpha_{1}-\alpha_{2}+\alpha_{3}-\alpha_{4}\right) \\
H 2=-H 3-\sqrt{2} R\left(\beta_{1}+\beta_{2}+\beta_{3}+\beta_{4}\right)
\end{gathered}
$$

The above relationship of the movement parameters can be varied in the alignment procedures. According to the actual mirror alignments presented and simulated, a flat mirror is put on M3 and calculates the value of $\mathrm{T} 2$ and $\mathrm{H} 2$ such that the ray passes the center of diaphragm. Then put, a spherical mirror on M3 back and calculate the proper location of T3 and H3

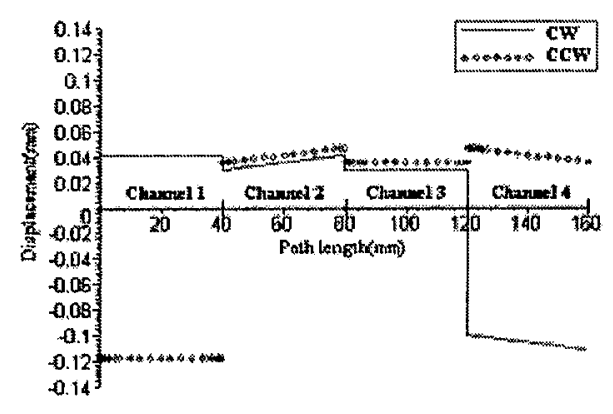

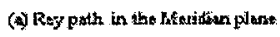

(a)

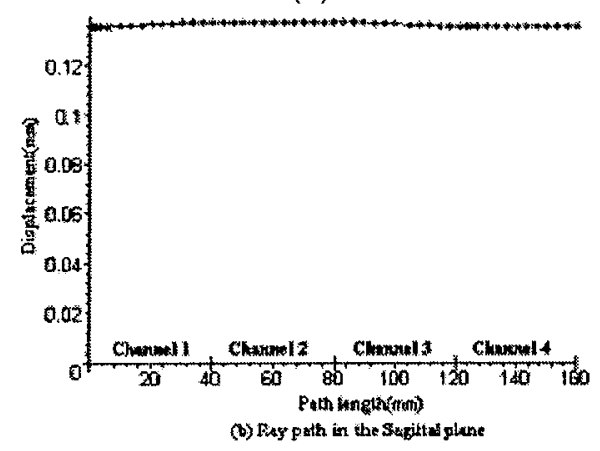

(b)

FIG. 5. Ray path in a $16 \mathrm{~cm}$ ring resonator with errors in Table 3. Here T2.H2. T3, and H3 are zero. in a similar way. The used relationships of the movement parameters are formulated as follows.

\section{CONCLUSION}

We showed that the $3 \times 3$ formulation of ray transfer matrix in the optical resonator is a very powerful tool in dealing with the effects of misalignment of

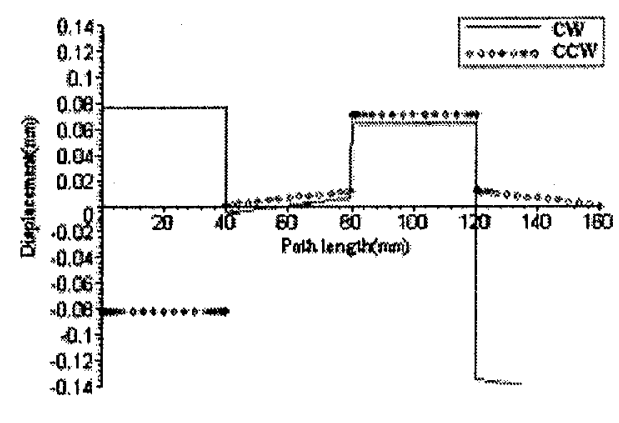

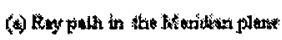

(a)

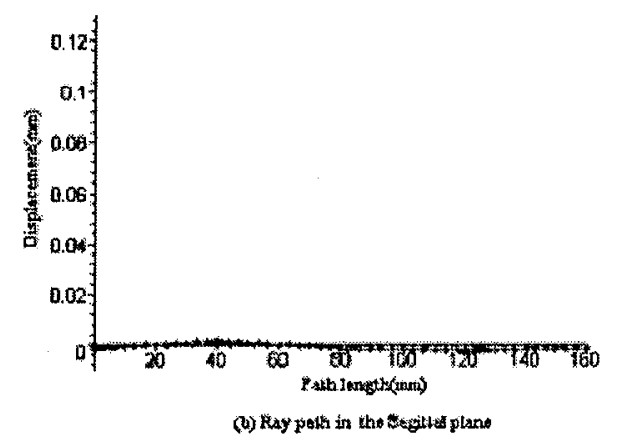

(b)

FIG. 6. Ray path in a $16 \mathrm{~cm}$ ring resonator after mirror alignments. 
optical components. Once monoblocks are manufactured, this tool can be used to predict a ray trajectory before or after the proper alignment as well as alignment sensitivity. Moreover, one could specify the manufacturing tolerance for the monoblock as required. Using this tool, we showed that the two counter-propagating beams do not take the same path, when the monoblock has manufacturing errors. Through the simulations, the effect of numerical adjustment movement can conceptually be verified. And the relationships of adjustment factors, Eq. (21), can present the guideline of movement in the actual adjustment situations. This $3 \times 3$ matrix formalism can be easily applied in a ring resonator with 3 mirrors.

\section{ACKNOWLEDGEMENT}

This study is supported by Korean Ministry of Science \& Technology through National Research Labo- ratory Program (No. 2000-N-NL-01-C-253)

*Corresponding author : dclee@iae.re.kr.

\section{REFERENCES}

[1] Ishchenko E.F., Journal of Applied Spectroscopy, 11, 456 (1969).

[2] A. Garrard and J. M. Burch, Introduction to Matrix Methods in Optics (John Wiley \& Sons, 1975).

[3] Joseph T. Verdeyen, Laser Electronics (Prentice-Hall Inc., 1981).

[4] Anthony E. Sigman, Lasers (University Science Books, 1986). 